\title{
On a reverse Mulholland-type inequality in the whole plane with general homogeneous kernel
}

Ricai Luo ${ }^{1 *}$, Bicheng Yang ${ }^{2}$ and Xingshou Huang ${ }^{1}$

"Correspondence:

hcxylor@126.com

${ }^{1}$ School of Mathematics and

Statistics, Hechi University, Yizhou, Guangxi 456300, P.R. China

Full list of author information is

available at the end of the article

\begin{abstract}
By using the idea of introducing parameters and weight coefficients, a new reverse discrete Mulholland-type inequality in the whole plane with general homogeneous kernel is given, which is an extension of the reverse Mulholland inequality. The equivalent forms are obtained. The equivalent statements of the best possible constant factor related to several parameters and a few applied examples are presented.
\end{abstract}

MSC: 26D15

Keywords: Weight coefficient; Mulholland-type inequality; Equivalent form; Equivalent statement; Parameter; Reverse

\section{Introduction}

Assuming that $p>1, \frac{1}{p}+\frac{1}{q}=1, a_{m}, b_{n} \geq 0,0<\sum_{m=1}^{\infty} a_{m}^{p}<\infty$ and $0<\sum_{n=1}^{\infty} b_{n}^{q}<\infty$, we have the following well-known Hardy-Hilbert inequality with the best possible constant $\frac{\pi}{\sin (\pi / p)}$ (cf. [1], Theorem 315):

$$
\sum_{m=1}^{\infty} \sum_{n=1}^{\infty} \frac{a_{m} b_{n}}{m+n}<\frac{\pi}{\sin (\pi / p)}\left(\sum_{m=1}^{\infty} a_{m}^{p}\right)^{\frac{1}{p}}\left(\sum_{n=1}^{\infty} b_{n}^{q}\right)^{\frac{1}{q}} .
$$

With regards to the similar assumption to (1), we still have Mulholland's inequality with the same best possible constant factor as follows (cf. [1], Theorem 343, replacing $\frac{a_{m}}{m}, \frac{b_{n}}{n}$ by $\left.a_{m}, b_{n}\right)$ :

$$
\sum_{m=2}^{\infty} \sum_{n=2}^{\infty} \frac{a_{m} b_{n}}{\ln m n}<\frac{\pi}{\sin (\pi / p)}\left(\sum_{m=2}^{\infty} \frac{a_{m}^{p}}{m^{1-p}}\right)^{1 / p}\left(\sum_{n=2}^{\infty} \frac{b_{n}^{q}}{n^{1-q}}\right)^{1 / q} .
$$

\section{Springer}

(c) The Author(s) 2021. This article is licensed under a Creative Commons Attribution 4.0 International License, which permits use, sharing, adaptation, distribution and reproduction in any medium or format, as long as you give appropriate credit to the original author(s) and the source, provide a link to the Creative Commons licence, and indicate if changes were made. The images or other third party material in this article are included in the article's Creative Commons licence, unless indicated otherwise in a credit line to the material. If material is not included in the article's Creative Commons licence and your intended use is not permitted by statutory regulation or exceeds the permitted use, you will need to obtain permission directly from the copyright holder. To view a copy of this licence, visit http://creativecommons.org/licenses/by/4.0/. 
If $f(x), g(y) \geq 0,0<\int_{0}^{\infty} f^{p}(x) d x<\infty$ and $0<\int_{0}^{\infty} g^{q}(y) d y<\infty$, then we have the integral analogous to (1), called Hardy-Hilbert's integral inequality, as follows:

$$
\int_{0}^{\infty} \int_{0}^{\infty} \frac{f(x) g(y)}{x+y} d x d y<\frac{\pi}{\sin (\pi / p)}\left(\int_{0}^{\infty} f^{p}(x) d x\right)^{\frac{1}{p}}\left(\int_{0}^{\infty} g^{q}(y) d y\right)^{\frac{1}{q}},
$$

where the constant factor $\frac{\pi}{\sin (\pi / p)}$ is still the best possible (cf. [1], Theorem 316).

In 1998, by introducing an independent parameter $\lambda>0$, Yang $[2,3]$ gave an extension of (2) (for $p=q=2$ ) with the kernel as $\frac{1}{(x+y)^{\lambda}}$ and a best possible constant factor $B\left(\frac{\lambda}{2}, \frac{\lambda}{2}\right)$ $(B(u, v)(u, v>0)$ is the beta function). Inequalities (1), (2) and (3) with their extensions and reverses play an important role in analysis and its applications (cf. [4-18]).

The following half-discrete Hilbert-type inequality was presented in 1934 (cf. [1], Theorem 351): If $K(x)(x>0)$ is decreasing, $p>1, \frac{1}{p}+\frac{1}{q}=1,0<\phi(s)=\int_{0}^{\infty} K(x) x^{s-1} d x<\infty$, then, for $a_{n} \geq 0,0<\sum_{n=1}^{\infty} a_{n}^{p}<\infty$,

$$
\int_{0}^{\infty} x^{p-2}\left(\sum_{n=1}^{\infty} K(n x) a_{n}\right)^{p} d x<\phi^{p}\left(\frac{1}{q}\right) \sum_{n=1}^{\infty} a_{n}^{p} .
$$

Some new extensions of (4) with the reverses were obtained by [19-24] in recent years.

In 2016, by using the technique of real analysis and the weight coefficients, Hong et al. [25] considered some equivalent statements of the extensions of (1) with the best possible constant factor related to several parameters. Other similar results about the extensions and the reverses of (1)-(4) were given by [26-46].

In this paper, following [25], by means of the idea of introducing parameters and the weight coefficients, a reverse discrete Mulholland-type in the whole plane is given as follows: for $r>1, \frac{1}{r}+\frac{1}{s}=1$,

$$
\begin{aligned}
& \sum_{|n|=3}^{\infty} \sum_{|m|=3}^{\infty} \frac{a_{m} b_{n}}{\ln |m n|} \\
& >\frac{2 \pi}{\sin (\pi / r)}\left[\sum_{|m|=3}^{\infty}\left(1-\tilde{\theta}_{1}\left(\frac{1}{s}, m\right)\right) \frac{\ln \frac{n^{\frac{p}{s}}-1}{|m|}}{|m|^{1-p}} a_{m}^{p}\right]^{\frac{1}{p}}\left(\sum_{|n|=3}^{\infty} \frac{\ln \frac{q}{r}-1|n|}{|n|^{1-q}} b_{n}^{q}\right)^{\frac{1}{q}},
\end{aligned}
$$

which is an extension of the reverse of (2). The general forms as well as the equivalent forms are obtained. The equivalent statements of the best possible constant factor related to several parameters are presented, and a few applied examples are considered.

\section{Some lemmas}

In what follows, we suppose that $0<p<1(q<0), \frac{1}{p}+\frac{1}{q}=1,-\frac{1}{2} \leq \alpha, \beta \leq \frac{1}{2}, \lambda, \lambda_{1}, \lambda_{2} \in R=$ $(-\infty, \infty), c:=\lambda-\lambda_{1}-\lambda_{2}, k_{\lambda}(x, y)(\geq 0)$ is a homogeneous function of degree $-\lambda$, satisfying

$$
k_{\lambda}(u x, u y)=u^{-\lambda} k_{\lambda}(x, y) \quad(u, x, y>0)
$$

and $k_{\lambda}(x, y) x^{\lambda_{1}-1}\left(\right.$ resp. $\left.k_{\lambda}(x, y) y^{\lambda_{2}-1}\right)$ is strictly decreasing with respect to $x>0$ (resp. $\left.y>0\right)$, such that

$$
k_{\lambda}(\gamma):=\int_{0}^{\infty} k_{\lambda}(1, u) u^{\gamma-1} d u \in R_{+}=(0, \infty) \quad\left(\gamma=\lambda_{2}, \lambda-\lambda_{1}\right) .
$$


We still assume that

$$
\theta_{\lambda}\left(\lambda_{2}, m\right):=\frac{1}{k_{\lambda}\left(\lambda_{2}\right)} \int_{0}^{\frac{2}{\ln (|m|+\alpha m)}} k_{\lambda}(1, u) u^{\lambda_{2}-1} d u \in(0,1),
$$

$a_{m}, b_{n} \geq 0(|m|,|n| \in N \backslash\{1,2\}=\{3,4, \ldots\})$, satisfying

$$
\begin{aligned}
& 0<\sum_{|m|=3}^{\infty} \frac{\ln ^{p\left(1-\lambda_{1}\right)-c-1}(|m|+\alpha m)}{(|m|+\alpha m)^{1-p}} a_{m}^{p}<\infty \quad \text { and } \\
& 0<\sum_{|n|=3}^{\infty} \frac{\ln ^{q\left(1-\lambda_{2}\right)-c-1}(|n|+\beta n)}{(|n|+\beta n)^{1-q}} b_{n}^{q}<\infty
\end{aligned}
$$

where $\sum_{|j|=3}^{\infty} \cdots=\sum_{j=-3}^{-\infty} \cdots+\sum_{j=3}^{\infty} \cdots(j=m, n)$

Lemma 1 For $\eta>0$, we have the following inequalities:

$$
\frac{2^{1-\eta}}{\eta\left(1-\beta^{2}\right)}<\sum_{|n|=3}^{\infty} \frac{\ln ^{-\eta-1}(|n|+\beta n)}{|n|+\beta n}<\frac{1}{\eta}\left(\eta O_{1}+\frac{2}{1-\beta^{2}}\right) .
$$

Proof In view of the decreasing property of series, for $\frac{e}{1 \pm \beta}<6,3<\frac{e^{2}}{1 \pm \beta}$, we have

$$
\begin{aligned}
\sum_{|n|=3}^{\infty} & \frac{\ln ^{-\eta-1}(|n|+\beta n)}{|n|+\beta n} \\
& =\sum_{n=-3}^{-\infty} \frac{\ln ^{-\eta-1}[(1-\beta)(-n)]}{(1-\beta)(-n)}+\sum_{n=3}^{\infty} \frac{\ln ^{-\eta-1}[(1+\beta) n]}{(1+\beta) n} \\
& =O_{1}+\sum_{n=7}^{\infty} \frac{\ln ^{-\eta-1}[(1-\beta) n]}{(1-\beta) n}+\sum_{n=7}^{\infty} \frac{\ln ^{-\eta-1}[(1+\beta) n]}{(1+\beta) n} \\
& <O_{1}+\int_{\frac{e}{1-\beta}}^{\infty} \frac{\ln ^{-\eta-1}[(1-\beta) y]}{(1-\beta) y} d y+\int_{\frac{e}{1+\beta}}^{\infty} \frac{\ln ^{-\eta-1}[(1+\beta) y]}{(1+\beta) y} d y \\
& =O_{1}+\frac{1}{\eta(1-\beta)}+\frac{1}{\eta(1+\beta)}=\frac{1}{\eta}\left(\eta O_{1}+\frac{2}{1-\beta^{2}}\right), \\
\sum_{|n|=3}^{\infty} & \frac{\ln ^{-\eta-1}(|n|+\beta n)}{|n|+\beta n} \\
& =\sum_{n=3}^{\infty} \frac{\ln ^{-\eta-1}[(1-\beta) n]}{(1-\beta) n}+\sum_{n=3}^{\infty} \frac{\ln ^{-\eta-1}[(1+\beta) n]}{(1+\beta) n} \\
& >\int_{\frac{e^{2}}{1-\beta}}^{\infty} \frac{\ln ^{-\eta-1}[(1-\beta) y]}{(1-\beta) y} d y+\int_{\frac{e^{2}}{1+\beta}}^{\infty} \frac{\ln ^{-\eta-1}[(1+\beta) y]}{(1+\beta) y} d y \\
& =\frac{2^{-\eta}}{\eta(1-\beta)}+\frac{2^{-\eta}}{\eta(1+\beta)}=\frac{2^{1-\eta}}{\eta\left(1-\beta^{2}\right)} .
\end{aligned}
$$

Hence, we have (7).

The lemma is proved. 
Definition 1 We set

$$
K(m, n):=k_{\lambda}(\ln (|m|+\alpha m), \ln (|n|+\beta n)) \quad(|m|,|n| \in N \backslash\{1,2\})
$$

and define the following weight coefficients:

$$
\begin{array}{ll}
\omega\left(\lambda_{2}, m\right):=\ln ^{\lambda-\lambda_{2}}(|m|+\alpha m) \sum_{|n|=3}^{\infty} K(m, n) \frac{\ln ^{\lambda_{2}-1}(|n|+\beta n)}{|n|+\beta n} \quad(|m| \in N \backslash\{1,2\}), \\
\varpi\left(\lambda_{1}, n\right):=\ln ^{\lambda-\lambda_{1}}(|n|+\beta n) \sum_{|m|=3}^{\infty} K(m, n) \frac{\ln ^{\lambda_{1}-1}(|m|+\alpha m)}{|m|+\alpha m} \quad(|n| \in N \backslash\{1,2\}) .
\end{array}
$$

Lemma 2 The following inequalities are valid:

$$
0<\frac{2 k_{\lambda}\left(\lambda_{2}\right)}{1-\beta^{2}}\left(1-\theta_{\lambda}\left(\lambda_{2}, m\right)\right)<\omega\left(\lambda_{2}, m\right)<\frac{2 k_{\lambda}\left(\lambda_{2}\right)}{1-\beta^{2}} \quad(|m| \in N \backslash\{1,2\}) .
$$

Proof For $|m| \in N \backslash\{1,2\}$, we set

$$
\begin{aligned}
& K^{(1)}(m, y):=k_{\lambda}(\ln (|m|+\alpha m), \ln [(1-\beta)(-y)]), \quad y<-\frac{1}{1-\beta}, \\
& K^{(2)}(m, y):=k_{\lambda}(\ln (|m|+\alpha m), \ln [(1+\beta) y]), \quad y>\frac{1}{1+\beta},
\end{aligned}
$$

where, from for $y>\frac{1}{1-\beta}, K^{(1)}(m,-y)=k_{\lambda}(\ln (|m|+\alpha m), \ln [(1-\beta) y])$. We find

$$
\begin{aligned}
\omega\left(\lambda_{2}, m\right)= & \ln ^{\lambda-\lambda_{2}}(|m|+\alpha m)\left\{\sum_{n=-3}^{-\infty} K^{(1)}(m, n) \frac{\ln ^{\lambda_{2}-1}[(1-\beta)(-n)]}{(1-\beta)(-n)}\right. \\
& \left.+\sum_{n=3}^{\infty} K^{(2)}(m, n) \frac{\ln ^{\lambda_{2}-1}[(1+\beta) n]}{(1+\beta) n}\right\} \\
= & \ln ^{\lambda-\lambda_{2}}(|m|+\alpha m)\left\{\sum_{n=3}^{\infty} K^{(1)}(m,-n) \frac{\ln ^{\lambda_{2}-1}[(1-\beta) n]}{(1-\beta) n}\right. \\
& \left.+\sum_{n=3}^{\infty} K^{(2)}(m, n) \frac{\ln ^{\lambda_{2}-1}[(1+\beta) n]}{(1+\beta) n}\right\} .
\end{aligned}
$$

It is evident that, for fixed $|m| \in N \backslash\{1,2\}$, by the assumptions, both

$$
K^{(1)}(m,-y) \frac{\ln ^{\lambda_{2}-1}[(1-\beta) y]}{(1-\beta) y}=\frac{1}{(1-\beta) y} k_{\lambda}(\ln (|m|+\alpha m), \ln [(1-\beta) y]) \ln ^{\lambda_{2}-1}[(1-\beta) y]
$$

and $K^{(1)}(m, y) \frac{\ln ^{\lambda} 2-1}{(1+\beta) y}$ are strictly decreasing with respect to $y>2$. By the decreasing property of series, we have

$$
\begin{aligned}
\omega\left(\lambda_{2}, m\right)< & \ln \lambda^{\lambda-\lambda_{2}}(|m|+\alpha m)\left\{\int _ { 2 } ^ { \infty } k _ { \lambda } \left(\ln (|m|+\alpha m, \ln [(1-\beta) y]) \frac{\ln ^{\lambda_{2}-1}[(1-\beta) y]}{(1-\beta) y} d y\right.\right. \\
& +\int_{2}^{\infty} k_{\lambda}\left(\ln (|m|+\alpha m, \ln [(1+\beta) y]) \frac{\ln ^{\lambda_{2}-1}[(1+\beta) y]}{(1+\beta) y} d y\right\}
\end{aligned}
$$




$$
\begin{aligned}
\omega\left(\lambda_{2}, m\right)> & \ln ^{\lambda-\lambda_{2}}(|m|+\alpha m)\left\{\int _ { 3 } ^ { \infty } k _ { \lambda } \left(\ln (|m|+\alpha m, \ln [(1-\beta) y]) \frac{\ln ^{\lambda_{2}-1}[(1-\beta) y]}{(1-\beta) y} d y\right.\right. \\
& +\int_{3}^{\infty} k_{\lambda}\left(\ln (|m|+\alpha m, \ln [(1+\beta) y]) \frac{\ln ^{\lambda_{2}-1}[(1+\beta) y]}{(1+\beta) y} d y\right\} .
\end{aligned}
$$

Setting $u=\frac{\ln [(1-\beta) y]}{\ln [|m|+\alpha m]}$ (resp. $\left.u=\frac{\ln [(1+\beta) y]}{\ln (|m|+\alpha m)}\right)$ in the above first (resp. second) integrals, since $2(1 \pm \beta) \geq 1$ and $3(1 \pm \beta)<e^{2}\left(\beta \in\left[-\frac{1}{2}, \frac{1}{2}\right]\right)$, we obtain

$$
\begin{aligned}
\omega\left(\lambda_{2}, m\right) & <\left[(1-\beta)^{-1}+(1+\beta)^{-1}\right] \int_{0}^{\infty} k_{\lambda}(1, u) u^{\lambda_{2}-1} d u=\frac{2 k_{\lambda}\left(\lambda_{2}\right)}{1-\beta^{2}}, \\
\omega\left(\lambda_{2}, m\right) & >\frac{1}{1-\beta} \int_{\frac{\ln [3(1-\beta)]}{\ln (|m|+\alpha m)}}^{\infty} k_{\lambda}(1, u) u^{\lambda_{2}-1} d u+\frac{1}{1+\beta} \int_{\frac{\ln [3(1+\beta)]}{\ln (|m|+\alpha m)}}^{\infty} k_{\lambda}(1, u) u^{\lambda_{2}-1} d u \\
& \geq \frac{2}{1-\beta^{2}} \int_{\frac{2}{\ln (|m|+\alpha m)}}^{\infty} k_{\lambda}(1, u) u^{\lambda_{2}-1} d u \\
& =\frac{2 k_{\lambda}\left(\lambda_{2}\right)}{1-\beta^{2}}\left(1-\theta_{\lambda}\left(\lambda_{2}, m\right)\right)>0 .
\end{aligned}
$$

Hence, we have (11).

The lemma is proved.

Note In the same way, we still have the following inequality:

$$
\varpi\left(\lambda_{1}, n\right)<\frac{2}{1-\alpha^{2}} k_{\lambda}\left(\lambda-\lambda_{1}\right) \quad(|n| \in N \backslash\{1,2\}) .
$$

Lemma 3 The following reverse Mulholland-type inequality in the whole plane is valid:

$$
\begin{aligned}
I:= & \sum_{|n|=3}^{\infty} \sum_{|m|=3}^{\infty} K(m, n) a_{m} b_{n} \\
> & \frac{2 k_{\lambda}^{\frac{1}{p}}\left(\lambda_{2}\right) k_{\lambda}^{\frac{1}{q}}\left(\lambda-\lambda_{1}\right)}{\left(1-\beta^{2}\right)^{1 / p}\left(1-\alpha^{2}\right)^{1 / q}}\left[\sum_{|m|=3}^{\infty}\left(1-\theta_{\lambda}\left(\lambda_{2}, m\right)\right) \frac{\ln ^{p\left(1-\lambda_{1}\right)-c-1}(|m|+\alpha m)}{(|m|+\alpha m)^{1-p}} a_{m}^{p}\right]^{\frac{1}{p}} \\
& \times\left[\sum_{|n|=3}^{\infty} \frac{\ln ^{q\left(1-\lambda_{2}\right)-c-1}(|n|+\beta n)}{(|n|+\beta n)^{1-q}} b_{n}^{q}\right]^{\frac{1}{q}} .
\end{aligned}
$$

Proof By the reverse Hölder inequality with weight (cf. [47]), we obtain

$$
\begin{aligned}
I= & \sum_{|n|=3}^{\infty} \sum_{|m|=3}^{\infty} K(m, n)\left[\frac{(|m|+\alpha m)^{1 / q} \ln ^{\left(\lambda_{2}-1\right) / p}(|n|+\beta n)}{(|n|+\beta n)^{1 / p} \ln ^{\left(\lambda_{1}-1\right) / q}(|m|+\alpha m)} a_{m}\right] \\
& \times\left[\frac{(|n|+\beta n)^{1 / p} \ln ^{\left(\lambda_{1}-1\right) / q}(|m|+\alpha m)}{(|m|+\alpha m)^{1 / q} \ln ^{\left(\lambda_{2}-1\right) / p}(|n|+\beta n)} b_{n}\right] \\
\geq & {\left[\sum_{|m|=3}^{\infty} \sum_{|n|=3}^{\infty} K(m, n) \frac{(|m|+\alpha m)^{p-1} \ln ^{\lambda_{2}-1}(|n|+\beta n)}{(|n|+\beta n) \ln ^{\left(\lambda_{1}-1\right)(p-1)}(\mid m+\alpha m)} a_{m}^{p}\right]^{\frac{1}{p}} }
\end{aligned}
$$




$$
\begin{aligned}
& \times\left[\sum_{|n|=3}^{\infty} \sum_{|m|=3}^{\infty} K(m, n) \frac{(|n|+\beta n)^{q-1} \ln ^{\lambda_{1}-1}(|m|+\alpha m)}{(|m|+\alpha m) \ln ^{\left(\lambda_{2}-1\right)(q-1)}(|n|+\beta n)} b_{n}^{q}\right]^{\frac{1}{q}} \\
= & {\left[\sum_{|m|=3}^{\infty} \omega\left(\lambda_{2}, m\right) \frac{\ln ^{p\left(1-\lambda_{1}\right)-c-1}(|m|+\alpha m)}{(|m|+\alpha m)^{1-p}} a_{m}^{p}\right]^{\frac{1}{p}} } \\
& \times\left[\sum_{|n|=3}^{\infty} \varpi\left(\lambda_{1}, n\right) \frac{\ln ^{q\left(1-\lambda_{2}\right)-c-1}(|n|+\beta n)}{(|n|+\beta n)^{1-q}} b_{n}^{q}\right]^{\frac{1}{q}} .
\end{aligned}
$$

Then, by (10) and (11), for $0<p<1, q<0$, we have (12).

The lemma is proved.

Remark 1 (i) By (12), for $\lambda_{1}+\lambda_{2}=\lambda$ (or $c=0$ ), we find

$$
\begin{aligned}
& 0<\sum_{|m|=3}^{\infty} \frac{\ln ^{p\left(1-\lambda_{1}\right)-1}(|m|+\alpha m)}{(|m|+\alpha m)^{1-p}} a_{m}^{p}<\infty, \\
& 0<\sum_{|n|=3}^{\infty} \frac{\ln ^{q\left(1-\lambda_{2}\right)-1}(|n|+\beta n)}{(|n|+\beta n)^{1-q}} b_{n}^{q}<\infty,
\end{aligned}
$$

and the following Mulholland-type inequality in the whole plane:

$$
\begin{aligned}
& \sum_{|n|=3}^{\infty} \sum_{|m|=3}^{\infty} k_{\lambda}(\ln (|m|+\alpha m), \ln (|n|+\beta n)) a_{m} b_{n} \\
& >\frac{2 k_{\lambda}\left(\lambda_{2}\right)}{\left(1-\beta^{2}\right)^{1 / p}\left(1-\alpha^{2}\right)^{1 / q}}\left[\sum_{|m|=3}^{\infty}\left(1-\theta_{\lambda}\left(\lambda_{2}, m\right)\right) \frac{\ln ^{p\left(1-\lambda_{1}\right)-1}(|m|+\alpha m)}{(|m|+\alpha m)^{1-p}} a_{m}^{p}\right]^{\frac{1}{p}} \\
& \quad \times\left[\sum_{|n|=3}^{\infty} \frac{\ln ^{q\left(1-\lambda_{2}\right)-1}(|n|+\beta n)}{(|n|+\beta n)^{1-q}} b_{n}^{q}\right]^{\frac{1}{q}} .
\end{aligned}
$$

In particular, for $\alpha=\beta=0, \lambda=1, k_{1}(x, y)=\frac{1}{x+y}, \lambda_{1}=\frac{1}{r}, \lambda_{2}=\frac{1}{s}\left(r>1, \frac{1}{r}+\frac{1}{s}=1\right)$,

$$
\tilde{\theta}_{1}\left(\frac{1}{s}, m\right):=\frac{\sin (\pi / r)}{\pi} \int_{0}^{\frac{2}{\ln |m|}} \frac{1}{1+u} u^{-\frac{1}{r}} d u \in(0,1)
$$

(14) reduces to (5); for $\alpha=\beta=0, a_{-m}=a_{m}, b_{-n}=b_{n}(m, n \in N \backslash\{1,2\})$ in (14), we have

$$
\begin{aligned}
& \vartheta_{\lambda}\left(\lambda_{2}, m\right):=\frac{1}{k_{\lambda}\left(\lambda_{2}\right)} \int_{0}^{\frac{2}{\ln m}} k_{\lambda}(1, u) u^{\lambda_{2}-1} d u \in(0,1) \quad(m \in\{3,4, \ldots\}), \\
& \sum_{n=3}^{\infty} \sum_{m=3}^{\infty} k_{\lambda}(\ln m, \ln n) a_{m} b_{n} \\
& \quad>k_{\lambda}\left(\lambda_{2}\right)\left[\sum_{m=3}^{\infty}\left(1-\vartheta_{\lambda}\left(\lambda_{2}, m\right)\right) \frac{\ln ^{p\left(1-\lambda_{1}\right)-1} m}{m^{1-p}} a_{m}^{p}\right]^{\frac{1}{p}}\left[\sum_{n=3}^{\infty} \frac{\ln ^{q\left(1-\lambda_{2}\right)-1}}{n^{1-q}} b_{n}^{q}\right]^{\frac{1}{q}} .
\end{aligned}
$$


(ii) For $\alpha=\beta= \pm \frac{1}{2}$ in (13), we have the following Mulholland-type inequality in the whole plane:

$$
\begin{aligned}
& \sum_{|n|=3}^{\infty} \sum_{|m|=3}^{\infty} k_{\lambda}\left(\ln \left(|m| \pm \frac{1}{2} m\right), \ln \left(|n| \pm \frac{1}{2} n\right)\right) a_{m} b_{n} \\
& >\frac{8}{3} k_{\lambda}\left(\lambda_{2}\right)\left[\sum_{|m|=3}^{\infty}\left(1-\hat{\theta}_{\lambda}\left(\lambda_{2}, m\right)\right) \frac{\ln ^{p\left(1-\lambda_{1}\right)-1}\left(|m| \pm \frac{1}{2} m\right)}{\left(|m| \pm \frac{1}{2} m\right)^{1-p}} a_{m}^{p}\right]^{\frac{1}{p}} \\
& \times\left[\sum_{|n|=3}^{\infty} \frac{\ln ^{q\left(1-\lambda_{2}\right)-1}\left(|n| \pm \frac{1}{2} n\right)}{\left(|n| \pm \frac{1}{2} n\right)^{1-q}} b_{n}^{q}\right]^{\frac{1}{q}}
\end{aligned}
$$

where $\hat{\theta}_{\lambda}\left(\lambda_{2}, m\right):=\frac{1}{k_{\lambda}\left(\lambda_{2}\right)} \int_{0}^{\frac{2}{\ln \left(|m| \pm \frac{1}{2} m\right)}} k_{\lambda}(1, u) u^{\lambda_{2}-1} d u \in(0,1)$.

Lemma 4 If there exists a constant $\delta_{0}>0$, such that $k_{\lambda}\left(\lambda_{2} \pm \delta_{0}\right)<\infty$, then, for any $0<\delta<\delta_{0}$, we have $k_{\lambda}\left(\lambda_{2} \pm \delta\right)<\infty$, and

$$
k_{\lambda}\left(\lambda_{2} \pm \delta\right) \rightarrow k_{\lambda}\left(\lambda_{2}\right) \quad\left(\delta \rightarrow 0^{+}\right)
$$

Proof For any $0<\delta<\delta_{0}$, we have

$$
\begin{aligned}
k_{\lambda}\left(\lambda_{2} \pm \delta\right) & =\int_{0}^{1} k_{\lambda}(1, u) u^{\lambda_{2} \pm \delta-1} d u+\int_{1}^{\infty} k_{\lambda}(1, u) u^{\lambda_{2} \pm \delta-1} d u \\
& \leq \int_{0}^{1} k_{\lambda}(1, u) u^{\lambda_{2}-\delta_{0}-1} d u+\int_{1}^{\infty} k_{\lambda}(1, u) u^{\lambda_{2}+\delta_{0}-1} d u \\
& \leq k_{\lambda}\left(\lambda_{2}-\delta_{0}\right)+k_{\lambda}\left(\lambda_{2}+\delta_{0}\right)<\infty .
\end{aligned}
$$

We find

$$
\begin{array}{ll}
0 \leq k_{\lambda}(1, u) u^{\lambda_{2} \pm \delta-1} \leq F(u):=k_{\lambda}(1, u) u^{\lambda_{2}-\delta_{0}-1} & (u \in(0,1)), \\
0 \leq k_{\lambda}(1, u) u^{\lambda_{2} \pm \delta-1} \leq F(u):=k_{\lambda}(1, u) u^{\lambda_{2}+\delta_{0}-1} & (u \in[1, \infty)),
\end{array}
$$

and then

$$
\begin{aligned}
\int_{0}^{\infty} F(u) d u & =\int_{0}^{1} F(u) d u+\int_{1}^{\infty} F(u) d u \\
& \leq k_{\lambda}\left(\lambda_{2}-\delta_{0}\right)+k_{\lambda}\left(\lambda_{2}+\delta_{0}\right)<\infty
\end{aligned}
$$

By Lebesgue dominated convergence theorem (cf. [48]), it follows that

$$
\int_{0}^{\infty} k_{\lambda}(1, u) u^{\lambda_{2} \pm \delta-1} d u \rightarrow \int_{0}^{\infty} k_{\lambda}(1, u) u^{\lambda_{2}-1} d u \quad\left(\delta \rightarrow 0^{+}\right)
$$

The lemma is proved.

Lemma 5 If there exist constants $\sigma_{0}, \delta_{0}>0$, such that $\theta_{\lambda}\left(\lambda_{2}, m\right)=O\left(\frac{1}{\left.\ln ^{\sigma_{0}(|m|+\alpha m)}\right)}\right)$ and $k_{\lambda}\left(\lambda_{2} \pm \delta_{0}\right)<\infty$, then the constant factor $\frac{2 k_{\lambda}\left(\lambda_{2}\right)}{\left(1-\beta^{2}\right)^{1 / p}\left(1-\alpha^{2}\right)^{1 / q}}$ in (13) is the best possible. 
Proof For any $0<\varepsilon<p \delta_{0}$, we set

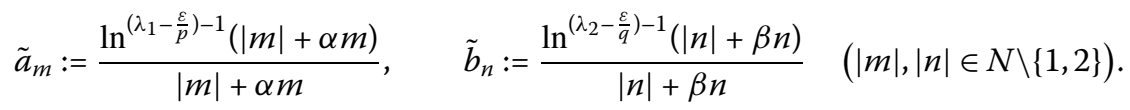

If there exists a constant $M\left(\geq \frac{2 k_{\lambda}\left(\lambda_{2}\right)}{\left(1-\beta^{2}\right)^{1 / p}\left(1-\alpha^{2}\right)^{1 / q}}\right)$, such that $(13)$ is valid when replacing $\frac{2 k_{\lambda}\left(\lambda_{2}\right)}{\left(1-\beta^{2}\right)^{1 / p}\left(1-\alpha^{2}\right)^{1 / q}}$ by $M$, then in particular, we have

$$
\begin{aligned}
\tilde{I}:= & \sum_{|n|=3}^{\infty} \sum_{|m|=3}^{\infty} K(m, n) \tilde{a}_{m} \tilde{b}_{n} \\
> & M\left[\sum_{|m|=3}^{\infty}\left(1-\theta_{\lambda}\left(\lambda_{2}, m\right)\right) \frac{\ln ^{p\left(1-\lambda_{1}\right)-1}(|m|+\alpha m)}{(|m|+\alpha m)^{1-p}} \tilde{a}_{m}^{p}\right]^{\frac{1}{p}} \\
& \times\left[\sum_{|n|=3}^{\infty} \frac{\ln ^{q\left(1-\lambda_{2}\right)-1}(|n|+\beta n)}{(|n|+\beta n)^{1-q}} \tilde{b}_{n}^{q}\right]^{\frac{1}{q}} .
\end{aligned}
$$

By the assumption, (10) and Lemma 1, we obtain

$$
\begin{aligned}
\tilde{I}> & M\left[\sum_{|m|=3}^{\infty}\left(1-O\left(\frac{1}{\ln ^{\sigma}(|m|+\alpha m)}\right)\right) \frac{\ln ^{-\varepsilon-1}(|m|+\alpha m)}{|m|+\alpha m}\right]^{\frac{1}{p}}\left[\sum_{|n|=3}^{\infty} \frac{\ln ^{-\varepsilon-1}(|n|+\beta n)}{|n|+\beta n}\right]^{\frac{1}{q}} \\
= & M\left[\sum_{|m|=3}^{\infty} \frac{\ln ^{-\varepsilon-1}(|m|+\alpha m)}{|m|+\alpha m}-\sum_{|m|=3}^{\infty} O\left(\frac{\ln ^{-\left(\sigma_{0}+\varepsilon\right)-1}(|m|+\alpha m)}{|m|+\alpha m}\right)\right]^{\frac{1}{p}} \\
& \times\left[\sum_{|n|=3}^{\infty} \frac{\ln ^{-\varepsilon-1}(|n|+\beta n)}{|n|+\beta n}\right]^{\frac{1}{q}} \\
> & \frac{M}{\varepsilon}\left(\frac{2^{1-\varepsilon}}{1-\alpha^{2}}-\varepsilon O(1)\right)^{\frac{1}{p}}\left(\varepsilon \tilde{O}_{1}+\frac{2}{1-\beta^{2}}\right)^{\frac{1}{q}} .
\end{aligned}
$$

By $(11)\left(\right.$ for $\left.\left(\lambda_{1}-\frac{\varepsilon}{p}\right)+\left(\lambda_{2}+\frac{\varepsilon}{p}\right)=\lambda\right)$ and Lemma 1 , since

$$
k_{\lambda}(x, y) x^{\lambda_{1}-\frac{\varepsilon}{p}-1}=\left(k_{\lambda}(x, y) x^{\lambda_{1}-1}\right) x^{-\frac{\varepsilon}{p}}
$$

is also strictly decreasing with respect to $x>0$, we obtain

$$
\begin{aligned}
\tilde{I} & =\sum_{|n|=3}^{\infty} \sum_{|m|=3}^{\infty} K(m, n) \frac{\ln ^{\left(\lambda_{1}-\frac{\varepsilon}{p}\right)-1}(|m|+\alpha m)}{|m|+\alpha m} \frac{\ln ^{\left(\lambda_{2}-\frac{\varepsilon}{q}\right)-1}(|n|+\beta n)}{|n|+\beta n} \\
& =\sum_{|n|=3}^{\infty} \varpi\left(\lambda_{1}-\frac{\varepsilon}{p}, n\right) \frac{\ln ^{-\varepsilon-1}(|n|+\beta n)}{|n|+\beta n}<\frac{2 k_{\lambda}\left(\lambda_{2}+\frac{\varepsilon}{p}\right)}{1-\alpha^{2}} \sum_{|n|=3}^{\infty} \frac{\ln ^{-\varepsilon-1}(|n|+\beta n)}{|n|+\beta n} \\
& <\frac{2 k_{\lambda}\left(\lambda_{2}+\frac{\varepsilon}{p}\right)}{1-\alpha^{2}}\left[O_{1}+\frac{2}{\varepsilon\left(1-\beta^{2}\right)}\right] .
\end{aligned}
$$


In view of the above results, we have

$$
\frac{2 k_{\lambda}\left(\lambda_{2}+\frac{\varepsilon}{p}\right)}{1-\alpha^{2}}\left(\varepsilon O_{1}+\frac{2}{1-\beta^{2}}\right)>\varepsilon \tilde{I}>M\left(\frac{2^{1-\varepsilon}}{1-\alpha^{2}}-\varepsilon O(1)\right)^{\frac{1}{p}}\left(\varepsilon \tilde{O}_{1}+\frac{2}{1-\beta^{2}}\right)^{\frac{1}{q}} .
$$

For $\varepsilon \rightarrow 0^{+}$, by Lemma 4 , we find $k_{\lambda}\left(\lambda_{2}+\frac{\varepsilon}{p}\right) \rightarrow k_{\lambda}\left(\lambda_{2}\right)$ and then

$$
\frac{4 k_{\lambda}\left(\lambda_{2}\right)}{\left(1-\alpha^{2}\right)\left(1-\beta^{2}\right)} \geq \frac{2 M}{\left(1-\alpha^{2}\right)^{1 / p}\left(1-\beta^{2}\right)^{1 / q}}
$$

namely, $\frac{2 k_{\lambda}\left(\lambda_{2}\right)}{\left(1-\beta^{2}\right)^{1 / p}\left(1-\alpha^{2}\right)^{1 / q}} \geq M$, which means that $M=\frac{2 k_{\lambda}\left(\lambda_{2}\right)}{\left(1-\beta^{2}\right)^{1 / p}\left(1-\alpha^{2}\right)^{1 / q}}$ is the best possible constant factor of (13).

The lemma is proved.

\section{Remark 2}

(i) Following the assumption of Lemma 5, the constant factors in (14) and (15) are also the best possible.

(ii) If there exists a constant $\delta_{0}>0$, such that $k_{\lambda}\left(\lambda_{2} \pm \delta_{0}\right)<\infty$, setting

$$
\hat{\lambda}_{1}:=\frac{\lambda-\lambda_{2}}{p}+\frac{\lambda_{1}}{q}=\lambda_{1}+\frac{c}{p}, \quad \hat{\lambda}_{2}:=\frac{\lambda-\lambda_{1}}{q}+\frac{\lambda_{2}}{p}=\lambda_{2}+\frac{c}{q},
$$

then we find $\hat{\lambda}_{1}+\hat{\lambda}_{2}=\lambda_{1}+\frac{c}{p}+\lambda_{2}+\frac{c}{q}=\lambda$, and for $c \in\left(-|q| \delta_{0},|q| \delta_{0}\right)$, by Lemma 4 and the reverse Hölder inequality (cf. [47]), we obtain

$$
\begin{aligned}
\infty & >k_{\lambda}\left(\hat{\lambda}_{2}\right) \\
& =k_{\lambda}\left(\frac{\lambda_{2}}{p}+\frac{\lambda-\lambda_{1}}{q}\right) \\
& =\int_{0}^{\infty} k_{\lambda}(1, u) u^{\frac{\lambda_{2}}{p}+\frac{\lambda-\lambda_{1}}{q}-1} d u \\
& =\int_{0}^{\infty} k_{\lambda}(1, u)\left(u^{\frac{\lambda_{2}-1}{p}}\right)\left(u^{\frac{\lambda-\lambda_{1}-1}{q}}\right) d u \\
& \geq\left(\int_{0}^{\infty} k_{\lambda}(1, u) u^{\lambda_{2}-1} d u\right)^{\frac{1}{p}}\left(\int_{0}^{\infty} k_{\lambda}(1, u) u^{\lambda-\lambda_{1}-1} d u\right)^{\frac{1}{q}} \\
& =k_{\lambda}^{\frac{1}{p}}\left(\lambda_{2}\right) k_{\lambda}^{\frac{1}{q}}\left(\lambda-\lambda_{1}\right)>0 .
\end{aligned}
$$

We can reduce (12) to the following:

$$
\begin{aligned}
I> & \frac{2 k_{\lambda}^{\frac{1}{p}}\left(\lambda_{2}\right) k_{\lambda}^{\frac{1}{q}}\left(\lambda-\lambda_{1}\right)}{\left(1-\beta^{2}\right)^{1 / p}\left(1-\alpha^{2}\right)^{1 / q}}\left[\sum_{|m|=3}^{\infty}\left(1-\theta_{\lambda}\left(\lambda_{2}, m\right)\right) \frac{\ln ^{p\left(1-\hat{\lambda}_{1}\right)-1}(|m|+\alpha m)}{(|m|+\alpha m)^{1-p}} a_{m}^{p}\right]^{\frac{1}{p}} \\
& \times\left[\sum_{|n|=3}^{\infty} \frac{\ln ^{q\left(1-\hat{\lambda}_{2}\right)-1}(|n|+\beta n)}{(|n|+\beta n)^{1-q}} b_{n}^{q}\right]^{\frac{1}{q}} \cdot
\end{aligned}
$$


Lemma 6 If there exists a constant $\delta_{0}>0$, such that $k_{\lambda}\left(\lambda_{2} \pm \delta_{0}\right)<\infty, c=\lambda-\lambda_{1}-\lambda_{2} \in$ $\left(-|q| \delta_{0},|q| \delta_{0}\right)$, and the constant factor $\frac{2 k_{\lambda}^{\frac{1}{p}}\left(\lambda_{2}\right) k_{\lambda}^{\frac{1}{q}}\left(\lambda-\lambda_{1}\right)}{\left(1-\beta^{2}\right)^{1 / p}\left(1-\alpha^{2}\right)^{1 / q}}$ in $(12)$ is the best possible, then we have $\lambda_{1}+\lambda_{2}=\lambda($ or $c=0)$.

Proof If the constant factor $\frac{2 k_{\lambda}^{\frac{1}{p}}\left(\lambda_{2}\right) k_{\lambda}^{\frac{1}{q}}\left(\lambda-\lambda_{1}\right)}{\left(1-\beta^{2}\right)^{1 / p}\left(1-\alpha^{2}\right)^{1 / q}}$ in (12) is the best possible, then, by (17) and (13) (for $\lambda_{i}=\hat{\lambda}_{i}(i=1,2)$ ), we have the following inequality:

$$
\frac{2 k_{\lambda}^{\frac{1}{p}}\left(\lambda_{2}\right) k_{\lambda}^{\frac{1}{q}}\left(\lambda-\lambda_{1}\right)}{\left(1-\beta^{2}\right)^{1 / p}\left(1-\alpha^{2}\right)^{1 / q}} \geq \frac{2 k_{\lambda}\left(\hat{\lambda}_{2}\right)}{\left(1-\beta^{2}\right)^{1 / p}\left(1-\alpha^{2}\right)^{1 / q}} \quad\left(\in R_{+}\right),
$$

namely, $k_{\lambda}^{\frac{1}{p}}\left(\lambda_{2}\right) k_{\lambda}^{\frac{1}{q}}\left(\lambda-\lambda_{1}\right) \geq k_{\lambda}\left(\hat{\lambda}_{2}\right)$, from which it follows that (17) keeps the form of equality.

We observe that (17) keeps the form of equality if and only if there exist constants $A$ and $B$, such that they are not both zero and (cf. [47])

$$
A u^{\lambda_{2}-1}=B u^{\lambda-\lambda_{1}-1} \quad \text { a.e. in } R_{+} .
$$

Assuming that $A \neq 0$, it follows that $u^{\lambda_{2}+\lambda_{1}-\lambda}=\frac{B}{A}$ a.e. in $R_{+}$, and then $\lambda_{2}+\lambda_{1}-\lambda=0$, namely, $\lambda_{1}+\lambda_{2}=\lambda$.

The lemma is proved.

\section{Main results}

Theorem 1 Inequality (12) is equivalent to the following reverse Mulholland-type inequalities in the whole plane:

$$
\begin{aligned}
J_{1} & :=\left[\sum_{|n|=3}^{\infty} \frac{\ln ^{p\left(\lambda_{2}+c\right)-c-1}(|n|+\beta n)}{|n|+\beta n}\left(\sum_{|m|=3}^{\infty} K(m, n) a_{m}\right)^{p}\right]^{\frac{1}{p}} \\
> & \frac{2 k_{\lambda}^{\frac{1}{p}}\left(\lambda_{2}\right) k_{\lambda}^{\frac{1}{q}}\left(\lambda-\lambda_{1}\right)}{\left(1-\beta^{2}\right)^{1 / p}\left(1-\alpha^{2}\right)^{1 / q}}\left[\sum_{|m|=3}^{\infty}\left(1-\theta_{\lambda}\left(\lambda_{2}, m\right)\right) \frac{\ln ^{p\left(1-\lambda_{1}\right)-c-1}(|m|+\alpha m)}{(|m|+\alpha m)^{1-p}} a_{m}^{p}\right]^{\frac{1}{p}}, \\
J_{2} & :=\left[\sum_{|m|=3}^{\infty} \frac{\ln ^{q\left(\lambda_{1}+c\right)-c-1}(|m|+\alpha m)}{(|m|+\alpha m)\left(1-\theta_{\lambda}\left(\lambda_{2}, m\right)\right)^{q-1}}\left(\sum_{|n|=3}^{\infty} K(m, n) b_{n}\right)^{q}\right]^{\frac{1}{q}} \\
& >\frac{2 k_{\lambda}^{\frac{1}{p}}\left(\lambda_{2}\right) k_{\lambda}^{\frac{1}{q}}\left(\lambda-\lambda_{1}\right)}{\left(1-\beta^{2}\right)^{1 / p}\left(1-\alpha^{2}\right)^{1 / q}}\left[\sum_{|n|=3}^{\infty} \frac{\ln ^{q\left(1-\lambda_{2}\right)-c-1}(|n|+\beta n)}{(|n|+\beta n)^{1-q}} b_{n}^{q}\right]^{\frac{1}{q}} .
\end{aligned}
$$

Proof Suppose that (19) is valid. By the reverse Hölder inequality (cf. [47]), we find

$$
\begin{aligned}
& I=\sum_{|n|=3}^{\infty}\left[\frac{\ln ^{\frac{-1}{p}+\lambda_{2}+\frac{c}{q}}(|n|+\beta n)}{(|n|+\beta n)^{1 / p}} \sum_{|m|=3}^{\infty} K(m, n) a_{m}\right]\left[\frac{\ln ^{\frac{1}{p}-\lambda_{2}-\frac{c}{q}}(|n|+\beta n)}{(|n|+\beta n)^{-1 / p}} b_{n}\right] \\
& \geq J_{1} \cdot\left[\sum_{|n|=3}^{\infty} \frac{\ln ^{q\left(1-\lambda_{2}\right)-c-1}(|n|+\beta n)}{(|n|+\beta n)^{1-q}} b_{n}^{q}\right]^{\frac{1}{q}} \text {. }
\end{aligned}
$$

Then, by (19), we obtain (13). 
On the other hand, assuming that (13) is valid, we set

$$
b_{n}:=\frac{\ln ^{p\left(\lambda_{2}+c\right)-c-1}(|n|+\beta n)}{|n|+\beta n}\left(\sum_{|m|=3}^{\infty} K(m, n) a_{m}\right)^{p-1}, \quad|n| \in N \backslash\{1,2\} .
$$

Then we have

$$
J_{1}^{p}=\sum_{|n|=3}^{\infty} \frac{\ln ^{q\left(1-\lambda_{2}\right)-c-1}(|n|+\beta n)}{(|n|+\beta n)^{1-q}} b_{n}^{q}=I
$$

If $J_{1}=0$, then (19) is naturally valid; if $J_{1}=\infty$, then it is impossible that makes (19) valid, namely, $J_{1}<\infty$. Suppose that $0<J_{1}<\infty$. By (13), it follows that

$$
\begin{aligned}
& \sum_{|n|=3}^{\infty} \frac{\ln ^{q\left(1-\lambda_{2}\right)-c-1}(|n|+\beta n)}{(|n|+\beta n)^{1-q}} b_{n}^{q} \\
& =J_{1}^{p}=I \\
& >\frac{2 k_{\lambda}^{\frac{1}{p}}\left(\lambda_{2}\right) k_{\lambda}^{\frac{1}{q}}\left(\lambda-\lambda_{1}\right)}{\left(1-\beta^{2}\right)^{1 / p}\left(1-\alpha^{2}\right)^{1 / q}}\left[\sum_{|m|=3}^{\infty}\left(1-\theta_{\lambda}\left(\lambda_{2}, m\right)\right) \frac{\ln ^{p\left(1-\lambda_{1}\right)-c-1}(|m|+\alpha m)}{(|m|+\alpha m)^{1-p}} a_{m}^{p}\right]^{\frac{1}{p}} J_{1}^{p-1}, \\
& J_{1}=\left[\sum_{|n|=3}^{\infty} \frac{\ln ^{q\left(1-\lambda_{2}\right)-c-1}(|n|+\beta n)}{(|n|+\beta n)^{1-q}} b_{n}^{q}\right]^{\frac{1}{p}} \\
& >\frac{2 k_{\lambda}^{\frac{1}{p}}\left(\lambda_{2}\right) k_{\lambda}^{\frac{1}{q}}\left(\lambda-\lambda_{1}\right)}{\left(1-\beta^{2}\right)^{1 / p}\left(1-\alpha^{2}\right)^{1 / q}}\left[\sum_{|m|=3}^{\infty}\left(1-\theta_{\lambda}\left(\lambda_{2}, m\right)\right) \frac{\ln ^{p\left(1-\lambda_{1}\right)-c-1}(|m|+\alpha m)}{(|m|+\alpha m)^{1-p}} a_{m}^{p}\right]^{\frac{1}{p}},
\end{aligned}
$$

namely, (19) follows, which is equivalent to (12).

Suppose that (20) is valid. By the reverse Hölder inequality (cf. [47]), we find

$$
\begin{aligned}
I= & \sum_{|m|=3}^{\infty}\left[\left(1-\theta_{\lambda}\left(\lambda_{2}, m\right)\right)^{\frac{1}{p}} \frac{\ln ^{\frac{1}{q}-\lambda_{1}-\frac{c}{p}}(|m|+\alpha m)}{(|m|+\alpha m)^{-1 / q}} a_{m}\right] \\
& \times\left[\frac{\ln ^{\frac{-1}{q}+\lambda_{1}+\frac{c}{p}}(|m|+\alpha m)}{(|m|+\alpha m)^{1 / q}\left(1-\theta_{\lambda}\left(\lambda_{2}, m\right)\right)^{1 / p}} \sum_{|n|=3}^{\infty} K(m, n) b_{n}\right] \\
\geq & {\left[\sum_{|m|=3}^{\infty}\left(1-\theta_{\lambda}\left(\lambda_{2}, m\right)\right) \frac{\ln ^{p\left(1-\lambda_{1}\right)-c-1}(|m|+\alpha m)}{(|m|+\alpha m)^{1-p}} a_{m}^{p}\right]^{\frac{1}{p}} J_{2} . }
\end{aligned}
$$

Then, by (20), we obtain (12).

On the other hand, assuming that (12) is valid, we set

$$
a_{m}:=\frac{\ln ^{q\left(\lambda_{1}+c\right)-c-1}(|m|+\alpha m)}{(|m|+\alpha m)\left(1-\theta_{\lambda}\left(\lambda_{2}, m\right)\right)^{q-1}}\left(\sum_{|m|=3}^{\infty} K(m, n) a_{m}\right)^{q-1}, \quad|m| \in N \backslash\{1,2\} .
$$


Then we have

$$
J_{2}^{q}=\sum_{|m|=3}^{\infty}\left(1-\theta_{\lambda}\left(\lambda_{2}, m\right)\right) \frac{\ln ^{p\left(1-\lambda_{1}\right)-c-1}(|m|+\alpha m)}{(|m|+\alpha m)^{1-p}} a_{m}^{p}=I .
$$

If $J_{2}=0$, then (20) is naturally valid; if $J_{2}=\infty$, then it is impossible that makes (20) valid, namely, $J_{2}<\infty$. Suppose that $0<J_{2}<\infty$. By (12), it follows that

$$
\begin{aligned}
& \sum_{|m|=3}^{\infty}\left(1-\theta_{\lambda}\left(\lambda_{2}, m\right)\right) \frac{\ln ^{p\left(1-\lambda_{1}\right)-c-1}(|m|+\alpha m)}{(|m|+\alpha m)^{1-p}} a_{m}^{p} \\
& =J_{2}^{q}=I \\
& >\frac{2 k_{\lambda}^{\frac{1}{p}}\left(\lambda_{2}\right) k_{\lambda}^{\frac{1}{q}}\left(\lambda-\lambda_{1}\right)}{\left(1-\beta^{2}\right)^{1 / p}\left(1-\alpha^{2}\right)^{1 / q}} J_{2}^{q-1} \cdot\left[\sum_{|n|=3}^{\infty} \frac{\ln ^{q\left(1-\lambda_{2}\right)-c-1}(|n|+\beta n)}{(|n|+\beta n)^{1-q}} b_{n}^{q}\right]^{\frac{1}{q}}, \\
& J_{2}=\left\{\sum_{|m|=3}^{\infty}\left(1-\theta_{\lambda}\left(\lambda_{2}, m\right)\right) \frac{\ln ^{p\left(1-\lambda_{1}\right)-c-1}(|m|+\alpha m)}{(|m|+\alpha m)^{1-p}} a_{m}^{p}\right\}^{\frac{1}{q}} \\
& >\frac{2 k_{\lambda}^{\frac{1}{p}}\left(\lambda_{2}\right) k_{\lambda}^{\frac{1}{q}}\left(\lambda-\lambda_{1}\right)}{\left(1-\beta^{2}\right)^{1 / p}\left(1-\alpha^{2}\right)^{1 / q}}\left[\sum_{|n|=3}^{\infty} \frac{\ln ^{q\left(1-\lambda_{2}\right)-c-1}(|n|+\beta n)}{(|n|+\beta n)^{1-q}} b_{n}^{q}\right]^{\frac{1}{q}},
\end{aligned}
$$

namely, (20) follows, which is equivalent to (12).

Hence, inequalities (12), (19) and (20) are equivalent.

The theorem is proved.

Theorem 2 Suppose that there exists a constant $\delta_{0}>0$, such that $k_{\lambda}\left(\lambda_{2} \pm \delta_{0}\right)<\infty$. The following statements (i), (ii), (iii), (iv), (v) and (vi) are equivalent:

(i) $\operatorname{Both} k_{\lambda}^{\frac{1}{p}}\left(\lambda_{2}\right) k_{\lambda}^{\frac{1}{q}}\left(\lambda-\lambda_{1}\right)$ and $k_{\lambda}\left(\frac{\lambda_{2}}{p}+\frac{\lambda-\lambda_{1}}{q}\right)$ are independent of $p, q$;

(ii)

$$
k_{\lambda}^{\frac{1}{p}}\left(\lambda_{2}\right) k_{\lambda}^{\frac{1}{q}}\left(\lambda-\lambda_{1}\right)=k_{\lambda}\left(\frac{\lambda_{2}}{p}+\frac{\lambda-\lambda_{1}}{q}\right)
$$

(iii) if $c \in\left(-|q| \delta_{0},|q| \delta_{0}\right)$, then $\lambda_{1}+\lambda_{2}=\lambda($ or $c=0)$;

(iv) if there exists a constant $\sigma_{0}>0$, such that $\theta_{\lambda}\left(\lambda_{2}, m\right)=O\left(\frac{1}{\ln ^{\sigma} 0(|m|+\alpha m)}\right)$, then $\frac{2 k_{\lambda}^{\frac{1}{p}}\left(\lambda_{2}\right) k_{\lambda}^{\frac{1}{q}}\left(\lambda-\lambda_{1}\right)}{\left(1-\beta^{2}\right)^{1 / p}\left(1-\alpha^{2}\right)^{1 / q}}$ is the best possible constant factor of $(12)$;

(v) $\frac{2 k_{\lambda}^{\frac{1}{p}}\left(\lambda_{2}\right) k_{\lambda}^{\frac{1}{q}}\left(\lambda-\lambda_{1}\right)}{\left(1-\beta^{2}\right)^{1 / p}\left(1-\alpha^{2}\right)^{1 / q}}$ is the best possible constant factor of $(19)$;

(vi) $\frac{2 k_{\lambda}^{\frac{1}{p}}\left(\lambda_{2}\right) k_{\lambda}^{\frac{1}{q}}\left(\lambda-\lambda_{1}\right)}{\left(1-\beta^{2}\right)^{1 / p}\left(1-\alpha^{2}\right)^{1 / q}}$ is the best possible constant factor of $(20)$.

If the statement (iii) follows, namely, $\lambda_{1}+\lambda_{2}=\lambda$ (or $c=0$ ), there exist constants $\sigma_{0}>0$, such that $\theta_{\lambda}\left(\lambda_{2}, m\right)=O\left(\frac{1}{\ln \sigma^{\sigma}(|m|+\alpha m)}\right)$, then we have the following equivalent inequalities 
equivalent to (13) with the best possible constant factor $\frac{2 k_{\lambda}\left(\lambda_{2}\right)}{\left(1-\beta^{2}\right)^{1 / p}\left(1-\alpha^{2}\right)^{1 / q}}$ :

$$
\begin{aligned}
& {\left[\sum_{|n|=3}^{\infty} \frac{\ln ^{p \lambda_{2}-1}(|n|+\beta n)}{|n|+\beta n}\left(\sum_{|m|=3}^{\infty} k_{\lambda}(\ln (|m|+\alpha m), \ln (|n|+\beta n)) a_{m}\right)^{p}\right]^{\frac{1}{p}}} \\
& \quad>\frac{2 k_{\lambda}\left(\lambda_{2}\right)}{\left(1-\beta^{2}\right)^{1 / p}\left(1-\alpha^{2}\right)^{1 / q}}\left[\sum_{|m|=3}^{\infty}\left(1-\theta_{\lambda}\left(\lambda_{2}, m\right)\right) \frac{\ln ^{p\left(1-\lambda_{1}\right)-1}(|m|+\alpha m)}{(|m|+\alpha m)^{1-p}} a_{m}^{p}\right]^{\frac{1}{p}}, \\
& {\left[\sum_{|m|=3}^{\infty} \frac{\ln ^{q \lambda_{1}-1}(|m|+\alpha m)}{(|m|+\alpha m)\left(1-\theta_{\lambda}\left(\lambda_{2}, m\right)\right)^{q-1}}\left(\sum_{|n|=3}^{\infty} k_{\lambda}(\ln (|m|+\alpha m), \ln (|n|+\beta n)) b_{n}\right)^{q}\right]^{\frac{1}{q}}} \\
& >\frac{2 k_{\lambda}\left(\lambda_{2}\right)}{\left(1-\beta^{2}\right)^{1 / p}\left(1-\alpha^{2}\right)^{1 / q}}\left[\sum_{|n|=3}^{\infty} \frac{\ln ^{q\left(1-\lambda_{2}\right)-1}(|n|+\beta n)}{(|n|+\beta n)^{1-q}} b_{n}^{q}\right]^{\frac{1}{q}} .
\end{aligned}
$$

In particular, for $\alpha=\beta=0, a_{-m}=a_{m}, b_{-n}=b_{n}(m, n \in N \backslash\{1,2\})$ in (25) and (26), we have the following inequalities equivalent to (14) with the best possible constant factor $k_{\lambda}\left(\lambda_{2}\right)$ :

$$
\begin{aligned}
& {\left[\sum_{n=3}^{\infty} \frac{\ln ^{p \lambda_{2}-1} n}{n}\left(\sum_{m=3}^{\infty} k_{\lambda}(\ln m, \ln n) a_{m}\right)^{p}\right]^{\frac{1}{p}}} \\
& >k_{\lambda}\left(\lambda_{2}\right)\left[\sum_{m=3}^{\infty}\left(1-\vartheta_{\lambda}\left(\lambda_{2}, m\right)\right) \frac{\ln ^{p\left(1-\lambda_{1}\right)-1} m}{m^{1-p}} a_{m}^{p}\right]^{\frac{1}{p}} \\
& {\left[\sum_{m=3}^{\infty} \frac{\ln ^{q \lambda_{1}-1} m}{m\left(1-\vartheta_{\lambda}\left(\lambda_{2}, m\right)\right)^{q-1}}\left(\sum_{n=3}^{\infty} k_{\lambda}(\ln m, \ln n) b_{n}\right)^{q}\right]^{\frac{1}{q}}} \\
& >k_{\lambda}\left(\lambda_{2}\right)\left[\sum_{n=3}^{\infty} \frac{\ln ^{q\left(1-\lambda_{2}\right)-1} n}{n^{1-q}} b_{n}^{q}\right]^{\frac{1}{q}} .
\end{aligned}
$$

Proof (i) $\Rightarrow$ (ii). Since $k_{\lambda}^{\frac{1}{p}}\left(\lambda_{2}\right) k_{\lambda}^{\frac{1}{q}}\left(\lambda-\lambda_{1}\right)$ is independent of $p, q$, we find

$$
k_{\lambda}^{\frac{1}{p}}\left(\lambda_{2}\right) k_{\lambda}^{\frac{1}{q}}\left(\lambda-\lambda_{1}\right)=\lim _{p \rightarrow 1^{-}} \lim _{q \rightarrow-\infty} k_{\lambda}^{\frac{1}{p}}\left(\lambda_{2}\right) k_{\lambda}^{\frac{1}{q}}\left(\lambda-\lambda_{1}\right)=k_{\lambda}\left(\lambda_{2}\right) .
$$

Then, by Lemma 4, we have the following equality:

$$
\begin{aligned}
k_{\lambda}\left(\frac{\lambda_{2}}{p}+\frac{\lambda-\lambda_{1}}{q}\right) & =k_{\lambda}\left(\lambda_{2}+\frac{c}{q}\right) \\
& =\lim _{q \rightarrow-\infty} k_{\lambda}\left(\lambda_{2}+\frac{c}{q}\right) \\
& =k_{\lambda}\left(\lambda_{2}\right)=k_{\lambda}^{\frac{1}{p}}\left(\lambda_{2}\right) k_{\lambda}^{\frac{1}{q}}\left(\lambda-\lambda_{1}\right) .
\end{aligned}
$$

(ii) $\Rightarrow$ (iii). If $k_{\lambda}^{\frac{1}{p}}\left(\lambda_{2}\right) k_{\lambda}^{\frac{1}{q}}\left(\lambda-\lambda_{1}\right)=k_{\lambda}\left(\frac{\lambda_{2}}{p}+\frac{\lambda-\lambda_{1}}{q}\right)$, then (17) keeps the form of an equality. By the proof of Lemma 6 , it follows that $\lambda_{1}+\lambda_{2}=\lambda$. 
(iii) $\Rightarrow$ (i). If $\lambda_{1}+\lambda_{2}=\lambda$, then we have

$$
k_{\lambda}\left(\frac{\lambda_{2}}{p}+\frac{\lambda-\lambda_{1}}{q}\right)=k_{\lambda}^{\frac{1}{p}}\left(\lambda_{2}\right) k_{\lambda}^{\frac{1}{q}}\left(\lambda-\lambda_{1}\right)=k_{\lambda}\left(\lambda_{2}\right) .
$$

Both $k_{\lambda}^{\frac{1}{p}}\left(\lambda_{2}\right) k_{\lambda}^{\frac{1}{q}}\left(\lambda-\lambda_{1}\right)$ and $k_{\lambda}\left(\frac{\lambda_{2}}{p}+\frac{\lambda-\lambda_{1}}{q}\right)$ are independent of $p, q$.

Hence, we have (i) $\Leftrightarrow$ (ii) $\Leftrightarrow$ (iii).

(iii) $\Leftrightarrow$ (iv). By Lemma 5 and Lemma 6, we obtain the conclusions.

(iv) $\Leftrightarrow$ (v). If the constant factor in (12) is the best possible, then so is constant factor in (19). Otherwise, by (21), we would arrive at a contradiction that the constant factor in (12) is not the best possible. On the other hand, if the constant factor in (19) is the best possible, then so is constant factor in (12). Otherwise, by (22), we would reach a contradiction that the constant factor in (19) is not the best possible.

(iv) $\Leftrightarrow$ (vi). If the constant factor in (12) is the best possible, then so is constant factor in (20). Otherwise, by (23), we would reach a contradiction that the constant factor in (12) is not the best possible. On the other hand, if the constant factor in (20) is the best possible, then so is constant factor in (12). Otherwise, by (24), we would reach a contradiction that the constant factor in (20) is not the best possible.

Therefore, the statements (i), (ii), (iii), (iv), (v) and (vi) are equivalent.

The theorem is proved.

\section{Some applied examples}

Example 1 For $\lambda>0, \lambda_{i} \in(0, \lambda) \cap(0,1](i=1,2)$, setting $k_{\lambda}(x, y)=\frac{1}{(x+y)^{\lambda}}(x, y>0)$, then $k_{\lambda}(x, y) x^{\lambda_{1}-1}$ (resp. $\left.k_{\lambda}(x, y) y^{\lambda_{2}-1}\right)$ is strictly decreasing with respect to $x>0$ (resp. $y>0$ ), such that (cf. [49])

$$
\begin{aligned}
& k_{\lambda}(\gamma)=\int_{0}^{\infty} \frac{u^{\gamma-1}}{(1+u)^{\lambda}} d u=B(\gamma \cdot \lambda-\gamma) \in R_{+} \quad\left(\gamma=\lambda_{2}, \lambda-\lambda_{1}\right), \\
& 0<\theta_{\lambda}\left(\lambda_{2}, m\right) \\
& \quad=\frac{1}{B\left(\lambda-\lambda_{2}, \lambda_{2}\right)} \int_{0}^{\frac{2}{\ln (|m|+\alpha m)}} \frac{u^{\lambda_{2}-1}}{(1+u)^{\lambda}} d u \\
& \leq \frac{1}{B\left(\lambda-\lambda_{2}, \lambda_{2}\right)} \int_{0}^{\frac{2}{\ln (|m|+\alpha m)}} u^{\lambda_{2}-1} d u \\
& =\frac{1}{\lambda_{2} B\left(\lambda-\lambda_{2}, \lambda_{2}\right)}\left(\frac{2}{\ln (|m|+\alpha m)}\right)^{\lambda_{2}} \quad\left(\sigma_{0}=\lambda_{2}>0\right) .
\end{aligned}
$$

Substitution of $K(m, n)=\frac{1}{\ln ^{\lambda}[(|m|+\alpha m)(|n|+\beta n)]}$ and

$$
k_{\lambda}^{\frac{1}{p}}\left(\lambda_{2}\right) k_{\lambda}^{\frac{1}{q}}\left(\lambda-\lambda_{1}\right)=\left(B\left(\lambda-\lambda_{2}, \lambda_{2}\right)\right)^{\frac{1}{p}}\left(B\left(\lambda_{1}, \lambda-\lambda_{1}\right)\right)^{\frac{1}{q}}
$$

in Lemma 3 and Theorem 1, we have the equivalent inequalities (12), (19) and (20) with the particular kernel as well as the particular constant factor. We set $\delta_{0}=\frac{1}{2} \min \left\{\lambda_{2}, \lambda-\lambda_{2}\right\}>0$, satisfying

$$
k_{\lambda}\left(\lambda_{2} \pm \delta_{0}\right)=B\left(\lambda-\lambda_{2} \mp \delta_{0}, \lambda_{2} \pm \delta_{0}\right) \in R_{+} .
$$


Then, by Theorem $2, \lambda_{1}+\lambda_{2}=\lambda$ if and only if the constant factor

$$
\frac{2 k_{\lambda}^{\frac{1}{p}}\left(\lambda_{2}\right) k_{\lambda}^{\frac{1}{q}}\left(\lambda-\lambda_{1}\right)}{\left(1-\beta^{2}\right)^{1 / p}\left(1-\alpha^{2}\right)^{1 / q}}\left(=\frac{2 B\left(\lambda_{1}, \lambda_{2}\right)}{\left(1-\beta^{2}\right)^{1 / p}\left(1-\alpha^{2}\right)^{1 / q}}\right)
$$

in (12), (19) and (20) is the best possible.

Example 2 For $\lambda>0, \lambda_{i} \in(0, \lambda) \cap(0,1](i=1,2)$, setting $k_{\lambda}(x, y)=\frac{\ln (x / y)}{x^{\lambda}-y^{\lambda}}(x, y>0)$, then $k_{\lambda}(x, y) x^{\lambda_{1}-1}$ (resp. $k_{\lambda}(x, y) y^{\lambda_{2}-1}$ ) is strictly decreasing with respect to $x>0$ (resp. $y>0$ ), such that

$$
k_{\lambda}(\gamma)=\int_{0}^{\infty} \frac{u^{\gamma-1} \ln u}{u^{\lambda}-1} d u=\left[\frac{\pi}{\lambda \sin (\pi \gamma / \lambda)}\right]^{2} \in R_{+} \quad\left(\gamma=\lambda_{2}, \lambda-\lambda_{1}\right) .
$$

For fixed $m$, since $f(u):=\frac{u^{\lambda} 2 / 2 \ln u}{u^{\lambda}-1}$ is continuous at $[0,2]\left(f(0):=0, f(1):=\frac{1}{\lambda}\right)$, we have

$$
\begin{aligned}
0 & <\theta_{\lambda}\left(\lambda_{2}, m\right) \\
& =\left[\frac{\lambda \sin \left(\pi \lambda_{2} / \lambda\right)}{\pi}\right]^{2} \int_{0}^{\frac{2}{\ln (|m|+\alpha m)}} \frac{u^{\lambda_{2}-1} \ln u}{u^{\lambda}-1} d u \\
& =\left[\frac{\lambda \sin \left(\pi \lambda_{2} / \lambda\right)}{\pi}\right]^{2} \int_{0}^{\frac{2}{\ln (|m|+\alpha m)}}\left(\frac{u^{\lambda / 2} \ln u}{u^{\lambda}-1}\right) u^{\frac{\lambda_{2}}{2}-1} d u \\
& \leq\left[\frac{\lambda \sin \left(\pi \lambda_{2} / \lambda\right)}{\pi}\right]^{2} M \int_{0}^{\frac{2}{\ln (|m|+\alpha m)}} u^{\frac{\lambda_{2}}{2}-1} d u \\
& =\left[\frac{\lambda \sin \left(\pi \lambda_{2} / \lambda\right)}{\pi}\right]^{2} \frac{2 M}{\lambda_{2}}\left(\frac{2}{\ln (|m|+\alpha m)}\right)^{\frac{\lambda_{2}}{2}} \quad\left(\sigma_{0}=\frac{\lambda_{2}}{2}>0\right) .
\end{aligned}
$$

Substitution of $K(m, n)=\frac{\ln [\ln (|m|+\alpha m) / \ln (|n|+\beta n)]}{\ln ^{\lambda}(|m|+\alpha m)-\ln ^{\lambda}(|n|+\beta n)}$ and

$$
k_{\lambda}^{\frac{1}{p}}\left(\lambda_{2}\right) k_{\lambda}^{\frac{1}{q}}\left(\lambda-\lambda_{1}\right)=\frac{1}{\lambda^{2}}\left[\frac{\pi}{\sin \left(\pi \lambda_{2} / \lambda\right)}\right]^{\frac{2}{p}}\left[\frac{\pi}{\sin \left(\pi \lambda_{1} / \lambda\right)}\right]^{\frac{2}{q}}
$$

in Lemma 3 and Theorem 1, we have equivalent reverse inequalities (12), (19) and (20) with the particular kernel as well as the particular constant factor. We set $\delta_{0}=\frac{1}{2} \min \left\{\lambda_{2}, \lambda-\lambda_{2}\right\}>$ 0 , satisfying

$$
k_{\lambda}\left(\lambda_{2} \pm \delta_{0}\right)=\left[\frac{\pi}{\lambda \sin \pi\left(\lambda_{2} \pm \delta_{0}\right) / \lambda}\right]^{2} \in R_{+} .
$$

Then, by Theorem $2, \lambda_{1}+\lambda_{2}=\lambda$ if and only if the constant factor

$$
\frac{2 k_{\lambda}^{\frac{1}{p}}\left(\lambda_{2}\right) k_{\lambda}^{\frac{1}{q}}\left(\lambda-\lambda_{1}\right)}{\left(1-\beta^{2}\right)^{1 / p}\left(1-\alpha^{2}\right)^{1 / q}}\left(=\frac{2\left[\frac{\pi}{\lambda \sin \left(\pi \lambda_{2} / \lambda\right)}\right]^{2}}{\left(1-\beta^{2}\right)^{1 / p}\left(1-\alpha^{2}\right)^{1 / q}}\right)
$$

in (12), (19) and (20) is the best possible. 
Example 3 For $0<\eta+\lambda_{i}<1(i=1,2), \lambda+2 \eta>\min _{i=1,2}\left\{0, \eta+\lambda_{i}\right\}$, setting $k_{\lambda}(x, y)=$ $\frac{(\min \{x, y\})^{\eta}}{(\max \{x, y\})^{\lambda+\eta}}(x, y>0)$, then

$$
k_{\lambda}(x, y) x^{\lambda_{1}-1}=\frac{(\min \{x, y\})^{\eta} x^{\lambda_{1}-1}}{(\max \{x, y\})^{\lambda+\eta}}= \begin{cases}x^{\eta+\lambda_{1}-1}, & 0<x<y \\ \frac{y^{\eta}}{x^{\lambda+\eta-\lambda_{1}+1}}, & x \geq y\end{cases}
$$

(resp. $k_{\lambda}(x, y) y^{\lambda_{2}-1}$ ) is strictly decreasing with respect to $x>0$ (resp. $y>0$ ), such that

$$
\begin{aligned}
& k_{\lambda}(\gamma)=\int_{0}^{\infty} \frac{(\min \{1, u\})^{\eta} u^{\gamma-1}}{(\max \{1, u\})^{\lambda+\eta}} d u \\
&=\int_{0}^{1} u^{\eta+\gamma-1} d u+\int_{1}^{\infty} \frac{u^{\gamma-1}}{u^{\lambda+\eta}} d u \\
&=\frac{\lambda+2 \eta}{(\eta+\gamma)(\lambda+\eta-\gamma)} \in R_{+} \quad\left(\gamma=\lambda_{2}, \lambda-\lambda_{1}\right), \\
& 0<\theta_{\lambda}\left(\lambda_{2}, m\right)=\frac{\left(\lambda_{2}+\eta\right)\left(\lambda-\lambda_{2}+\eta\right)}{\lambda+\eta} \int_{0}^{\frac{2}{\ln (|m|+\alpha m)}} \frac{(\min \{1, u\})^{\eta} u^{\lambda_{2}-1}}{(\max \{1, u\})^{\lambda+\eta}} d u \\
&=\frac{\left(\lambda_{2}+\eta\right)\left(\lambda-\lambda_{2}+\eta\right)}{\lambda+\eta} \int_{0}^{\frac{2}{\ln (|m|+\alpha m)}} u^{\eta+\lambda_{2}-1} d u \\
&=\frac{\lambda-\lambda_{2}+\eta}{\lambda+\eta}\left(\frac{2}{\ln (|m|+\alpha m)}\right)^{\eta+\lambda_{2}} \quad\left(\ln (|m|+\alpha m)>2 ; \sigma_{0}=\eta+\lambda_{2}>0\right) .
\end{aligned}
$$

Substitution of $K(m, n)=\frac{(\min \{\ln (|m|+\alpha m), \ln (|n|+\beta n)\})^{\eta}}{(\max \{\ln (|m|+\alpha m), \ln (|n|+\beta n)\})^{\lambda+\eta}}$ and

$$
k_{\lambda}^{\frac{1}{p}}\left(\lambda_{2}\right) k_{\lambda}^{\frac{1}{q}}\left(\lambda-\lambda_{1}\right)=\left[\frac{\lambda+2 \eta}{\left(\lambda_{2}+\eta\right)\left(\lambda-\lambda_{2}+\eta\right)}\right]^{\frac{2}{p}}\left[\frac{\lambda+2 \eta}{\left(\lambda_{1}+\eta\right)\left(\lambda-\lambda_{1}+\eta\right)}\right]^{\frac{2}{q}}
$$

in Lemma 3 and Theorem 1, we have equivalent reverse inequalities (12), (19) and (20) with the particular kernel as well as the particular constant factor. We set $\delta_{0}=\frac{1}{2} \min \{\eta+$ $\left.\lambda_{2}, \eta+\lambda-\lambda_{2}\right\}>0$, satisfying

$$
k_{\lambda}\left(\lambda_{2} \pm \delta_{0}\right)=\frac{\lambda+2 \eta}{\left(\eta+\lambda_{2} \pm \delta_{0}\right)\left(\eta+\lambda-\lambda_{2} \mp \delta_{0}\right)} \in R_{+}
$$

Then, by Theorem $2, \lambda_{1}+\lambda_{2}=\lambda$ if and only if the constant factor

$$
\frac{2 k_{\lambda}^{\frac{1}{p}}\left(\lambda_{2}\right) k_{\lambda}^{\frac{1}{q}}\left(\lambda-\lambda_{1}\right)}{\left(1-\beta^{2}\right)^{1 / p}\left(1-\alpha^{2}\right)^{1 / q}}\left(=\frac{2}{\left(1-\beta^{2}\right)^{1 / p}\left(1-\alpha^{2}\right)^{1 / q}} \frac{\lambda+2 \eta}{\left(\eta+\lambda_{2}\right)\left(\eta+\lambda-\lambda_{2}\right)}\right)
$$

in (12), (19) and (20) is the best possible.

\section{Example 4}

(i) In view of the following expression for the cotangent function (cf. [50]):

$$
\cot x=\frac{1}{x}+\sum_{k=1}^{\infty}\left(\frac{1}{x-\pi k}+\frac{1}{x+\pi k}\right) \quad(x \in(0, \pi))
$$


for $b \in(0,1)$, by the Lebesgue term by term theorem (cf. [44]), we obtain

$$
\begin{aligned}
A_{b} & :=\int_{0}^{\infty} \frac{u^{b-1}}{1-u} d u=\int_{0}^{1} \frac{u^{b-1}}{1-u} d u+\int_{1}^{\infty} \frac{u^{b-1}}{1-u} d u \\
& =\int_{0}^{1} \frac{u^{b-1}}{1-u} d u-\int_{0}^{1} \frac{v^{-b}}{1-v} d v=\int_{0}^{1} \frac{u^{b-1}-u^{-b}}{1-u} d u \\
& =\int_{0}^{1} \sum_{k=0}^{\infty}\left(u^{k+b-1}-u^{k-b}\right) d u=\sum_{k=0}^{\infty} \int_{0}^{1}\left(u^{k+b-1}-u^{k-b}\right) d u \\
& =\sum_{k=0}^{\infty}\left(\frac{1}{k+b}-\frac{1}{k+1-b}\right)=\pi\left[\frac{1}{\pi b}+\sum_{k=1}^{\infty}\left(\frac{1}{\pi b-\pi k}+\frac{1}{\pi b+\pi k}\right)\right] \\
& =\pi \cot \pi b \in R:=(-\infty, \infty)
\end{aligned}
$$

(ii) For $\lambda, \eta>0$, we set the homogeneous function of degree $-\lambda$ as follows:

$$
k_{\lambda}(x, y):=\frac{x^{\eta}-y^{\eta}}{x^{\lambda+\eta}-y^{\lambda+\eta}} \quad(x, y>0)
$$

satisfying $k_{\lambda}(v, v):=\frac{\eta}{(\lambda+\eta) v^{\lambda}}(v>0)$. It follows that $k_{\lambda}(x, y)$ is a positive and continuous function with respect to $x, y>0$. For $x \neq y$, we find

$$
\frac{\partial}{\partial x} k_{\lambda}(x, y)=-x^{\eta-1}\left(x^{\lambda+\eta}-y^{\lambda+\eta}\right)^{-2} \varphi(x, y)
$$

where we set the following differentiable function:

$$
\varphi(x, y):=\lambda x^{\lambda+\eta}-(\lambda+\eta) y^{\eta} x^{\lambda}+\eta y^{\lambda+\eta} \quad(x, y>0) .
$$

We find that, for $0<x<y$,

$$
\frac{\partial}{\partial x} \varphi(x, y)=\lambda(\lambda+\eta) x^{\lambda-1}\left(x^{\eta}-y^{\eta}\right)<0
$$

for $x>y, \frac{\partial}{\partial x} \varphi(x, y)>0$. It follows that $\varphi(x, y)$ is strictly decreasing (resp. increasing) with respect to $x<y$ (resp. $x>y)$. Since $\varphi(y, y)=\min _{x>0} \varphi(x, y)=0(y>0)$, we have $\varphi(x, y)>0(x \neq y)$, namely, $\frac{\partial}{\partial x} k_{\lambda}(x, y)<0(x \neq y)$. Therefore, in view of $k_{\lambda}(x, y)$ is continuous at $x=y$, we confirm that $k_{\lambda}(x, y)(y>0)$ is strictly decreasing with respect to $x>0$. In the same way, we can show that $k_{\lambda}(x, y)(x>0)$ is also strictly decreasing with respect to $y>0$. Hence, for $\lambda_{i} \in(0, \lambda) \cap(0,1](i=1,2), k_{\lambda}(x, y) x^{\lambda_{1}-1}$ (resp. $k_{\lambda}(x, y) y^{\lambda_{2}-1}$ ) is strictly decreasing with respect to $x>0$ (resp. $y>0$ ).

(iii) Since $k_{\lambda}(x, y)>0$, by (i), we obtain

$$
\begin{aligned}
k_{\lambda, \eta}(\gamma) & :=\int_{0}^{\infty} k_{\lambda}(1, u) u^{\gamma-1} d u=\int_{0}^{\infty} \frac{1-u^{\eta}}{1-u^{\lambda+\eta}} u^{\gamma-1} d u \\
& \stackrel{\nu=u^{\lambda+\eta}}{=} \frac{1}{\lambda+\eta}\left(\int_{0}^{\infty} \frac{v^{\frac{\gamma}{\lambda+\eta}}-1}{1-\nu} d v-\int_{0}^{\infty} \frac{v^{\frac{\gamma+\eta}{\lambda+\eta}-1}}{1-v} d v\right) \\
& =\frac{\pi}{\lambda+\eta}\left[\cot \left(\frac{\pi \gamma}{\lambda+\eta}\right)-\cot \left(\frac{\pi(\gamma+\eta)}{\lambda+\eta}\right)\right]
\end{aligned}
$$




$$
\begin{aligned}
& \quad=\frac{\pi}{\lambda+\eta}\left[\cot \left(\frac{\pi \gamma}{\lambda+\eta}\right)+\cot \left(\frac{\pi(\lambda-\gamma)}{\lambda+\eta}\right)\right] \in R_{+} \quad\left(\lambda=\lambda_{2}, \lambda-\lambda_{1}\right), \\
& 0<\theta_{\lambda}\left(\lambda_{2}, m\right) \\
& =\frac{1}{k_{\lambda, \eta}\left(\lambda_{2}\right)} \int_{0}^{\frac{2}{\ln (m \mid+\alpha m)}} \frac{\left(1-u^{\eta}\right) u^{\lambda_{2}-1}}{1-u^{\lambda+\eta}} d u \leq \frac{1}{k_{\lambda, \eta}\left(\lambda_{2}\right)} \int_{0}^{\frac{2}{\ln (m \mid+\alpha m)}} u^{\lambda_{2}-1} d u \\
& =\frac{1}{\lambda_{2} k_{\lambda, \eta}\left(\lambda_{2}\right)}\left(\frac{2}{\ln (|m|+\alpha m)}\right)^{\lambda_{2}}\left(\sigma_{0}=\lambda_{2}>0\right) .
\end{aligned}
$$

On substitution of $K(m, n)=\frac{\ln ^{\eta}(|m|+\alpha m)-\ln ^{\eta}(|n|+\beta n)}{\ln ^{\lambda+\eta}(|m|+\alpha m)-\ln ^{\lambda+\eta}(|n|+\beta n)}$ and

$$
k_{\lambda}^{\frac{1}{p}}\left(\lambda_{2}\right) k_{\lambda}^{\frac{1}{q}}\left(\lambda-\lambda_{1}\right)=k_{\lambda, \eta}^{\frac{1}{p}}\left(\lambda_{2}\right) k_{\lambda, \eta}^{\frac{1}{q}}\left(\lambda-\lambda_{1}\right)
$$

in Lemma 3 and Theorem 1, we have the equivalent inequalities (12), (19) and (20) with the particular kernel as well as the particular constant factor. We set $\delta_{0}=\frac{1}{2} \min \left\{\lambda_{2}, \lambda-\lambda_{2}\right\}>0$, satisfying $k_{\lambda, \eta}\left(\lambda_{2} \pm \delta_{0}\right) \in R_{+}$. Then, by Theorem $2, \lambda_{1}+\lambda_{2}=\lambda$ if and only if the constant factor

$$
\frac{2 k_{\lambda, \eta}^{\frac{1}{p}}\left(\lambda_{2}\right) k_{\lambda, \eta}^{\frac{1}{q}}\left(\lambda-\lambda_{1}\right)}{\left(1-\beta^{2}\right)^{1 / p}\left(1-\alpha^{2}\right)^{1 / q}}\left(=\frac{2 k_{\lambda, \eta}\left(\lambda_{2}\right)}{\left(1-\beta^{2}\right)^{1 / p}\left(1-\alpha^{2}\right)^{1 / q}}\right)
$$

in (13), (19) and (20) is the best possible.

\section{Conclusions}

In this paper, by means of the idea of introducing parameters and the weight coefficients, a new reverse discrete Mulholland-type inequality in the whole plane is obtained in Lemma 3, which is an extension of the reverse of (2). The equivalent forms are given in Theorem 1 . The equivalent statements of the best possible constant factor related to several parameters are considered in Theorem 2. Some particular inequalities are presented in Theorem 2 and Remark 1. Some applied examples are given in Example 1-4. The lemmas and theorems provide an extensive account of this type of inequalities.

\section{Acknowledgements}

The authors thank the referee for his useful proposal to amend the paper.

\section{Funding}

This work is supported by the National Science Foundation of China (11961021 and 11561019). Guangxi Natural Science Foundation (2020GXNSFAA159084 and 2020GXNSFAA159172), the Hechi University Research Fund for Advanced Talents (2019GCC005) and Characteristic innovation project of Guangdong Provincial Colleges and universities in 2020 (2020KTSCX088)

Availability of data and materials

We declare that the data and material in this paper can be used publicly.

\section{Competing interests}

The authors declare that they have no competing interests.

\section{Authors' contributions}

BY carried out the mathematical studies, participated in the sequence alignment and drafted the manuscript. RL and XH participated in the design of the study and performed the numerical analysis. All authors read and approved the final manuscript.

\section{Author details}

${ }^{1}$ School of Mathematics and Statistics, Hechi University, Yizhou, Guangxi 456300, P.R. China. ${ }^{2}$ Department of Mathematics, Guangdong University of Education, Guangzhou, Guangdong 51003, China. 


\section{Publisher's Note}

Springer Nature remains neutral with regard to jurisdictional claims in published maps and institutional affiliations.

\section{Received: 1 August 2020 Accepted: 25 February 2021 Published online: 06 March 2021}

\section{References}

1. Hardy, G.H., Littlewood, J.E., Polya, G.: Inequalities. Cambridge University Press, Cambridge (1934)

2. Yang, B.C.: On Hilbert's integral inequality. J. Math. Anal. Appl. 220, 778-785 (1998)

3. Yang, B.C.: A note on Hilbert's integral inequality. Chin. Q. J. Math. 13(4), 83-86 (1998)

4. Yang, B.C.: The Norm of Operator and Hilbert-Type Inequalities. Science Press, Beijing (2009)

5. Yang, B.C.: Hilbert-Type Integral Inequalities. Bentham Science Publishers, United Arab Emirates (2009)

6. Krnić, M., Pečarić, J.: General Hilbert's and Hardy's inequalities. Math. Inequal. Appl. 8, 29-51 (2005)

7. Perić, I., Vuković, P.: Multiple Hilbert's type inequalities with a homogeneous kernel. Banach J. Math. Anal. 5(2), 33-43 (2011)

8. Huang, Q.L:: A new extension of Hardy-Hilbert-type inequality. J. Inequal. Appl. 2015, 397 (2015)

9. He, B.: A multiple Hilbert-type discrete inequality with a new kernel and best possible constant factor. J. Appl. Math. Anal. Appl. 431, 889-902 (2015)

10. Xu, J.S.: Hardy-Hilbert's inequalities with two parameters. Adv. Math. 36(2), 63-76 (2007)

11. Xie, Z.T., Zeng, Z., Sun, Y.F.: A new Hilbert-type inequality with the homogeneous kernel of degree -2. Adv. Appl. Math. Sci. 12(7), 391-401 (2013)

12. Zhen, Z., Raja Rama Gandhi, K., Xie, Z.T.: A new Hilbert-type inequality with the homogeneous kernel of degree -2 and with the integral. Bull. Math. Sci. Appl. 3(1), 11-20 (2014)

13. Xin, D.M.: A Hilbert-type integral inequality with the homogeneous kernel of zero degree. Math. Theory Appl. 30(2), 70-74 (2010)

14. Azar, L.E.: The connection between Hilbert and Hardy inequalities. J. Inequal. Appl. 2013, 452 (2013)

15. Adiyasuren, V., Batbold, T., Krnić, M.: Hillbert-type inequalities involving differential operators, the best constants and applications. Math. Inequal. Appl. 18(1), 111-124 (2015)

16. Chen, Q., Yang, B.C.: A survey on the study of Hilbert-type inequalities. J. Inequal. Appl. 2015, 302 (2015)

17. Debnath, L., Yang, B.C.: Recent developments of Hilbert-type discrete and integral inequalities with applications. Int. J. Math. Math. Sci. 2012, Article ID 871845 (2012)

18. Mitrinovic, D.S., Pecaric, J.E., Fink, A.M.: Inequalities Involving Functions and Their Integrals and Derivatives. Kluwer Academic, Dordrecht (1991)

19. Rassias, M.T., Yang, B.C.: On half-discrete Hilbert's inequality. Appl. Math. Comput. 220, 75-93 (2013)

20. Yang, B.C., Krnic, M.: A half-discrete Hilbert-type inequality with a general homogeneous kernel of degree 0. J. Math. Inequal. 6(3), 401-417 (2012)

21. Rassias, M.T., Yang, B.C.: A multidimensional half-discrete Hilbert-type inequality and the Riemann zeta function. Appl. Math. Comput. 225, 263-277 (2013)

22. Rassias, M.T., Yang, B.C.: On a multidimensional half-discrete Hilbert-type inequality related to the hyperbolic cotangent function. Appl. Math. Comput. 242, 800-813 (2013)

23. Huang, Z.X., Yang, B.C.: On a half-discrete Hilbert-type inequality similar to Mulholland's inequality. J. Inequal. Appl. $2013,290(2013)$

24. Yang, B.C., Debnath, L.: Half-Discrete Hilbert-Type Inequalities. World Scientific, Singapore (2014)

25. Hong, Y., Wen, Y:: A necessary and sufficient condition of that Hilbert type series inequality with homogeneous kernel has the best constant factor. Ann. Math. 37A(3), 329-336 (2016)

26. Hong, Y.: On the structure character of Hilbert's type integral inequality with homogeneous kernel and applications. J. Jilin Univ. Sci. Ed. 55(2), 189-194 (2017)

27. Hong, Y., Huang, Q.L., Yang, B.C., Liao, J.Q.: The necessary and sufficient conditions for the existence of a kind of Hilbert-type multiple integral inequality with the non-homogeneous kernel and its applications. J. Inequal. Appl. 2017, $316(2017)$

28. Xin, D.M., Yang, B.C., Wang, A.Z.: Equivalent property of a Hilbert-type integral inequality related to the beta function in the whole plane. J. Funct. Spaces 2018, Article ID 2691816 (2018)

29. Hong, Y., He, B., Yang, B.C.: Necessary and sufficient conditions for the validity of Hilbert type integral inequalities with a class of quasi-homogeneous kernels and its application in operator theory. J. Math. Inequal. 12(3), 777-788 (2018)

30. Huang, Z.X., Yang, B.C.: Equivalent property of a half-discrete Hilbert's inequality with parameters. J. Inequal. Appl. 2018, $333(2018)$

31. He, L.P., Liu, H.Y., Yang, B.C.: Parametric Mulholland-type inequalities. J. Appl. Anal. Comput. 9(5), 1973-1986 (2019)

32. Yang, B.C., Hauang, M.F., Zhong, Y.R.: On an extended Hardy-Hilbert's inequality in the whole plane. J. Appl. Anal. Comput. 9(6), 2124-2136 (2019)

33. Yang, B.C., Wu, S., Wang, A.: On a reverse half-discrete Hardy-Hilbert's inequality with parameters. Mathematics 7, 1054 (2019)

34. Wang, A.Z., Yang, B.C., Chen, Q.: Equivalent properties of a reverse's half-discrete Hilbert's inequality. J. Inequal. Appl. 2019, $279(2019)$

35. Yang, B.C., Wu, S.H., Liao, J.Q.: On a new extended Hardy-Hilbert's inequality with parameters. Mathematics 8, 73 (2020). https://doi.org/10.3390/math8010073

36. Mo, H.M., Yang, B.C.: On a new Hilbert-type integral inequality involving the upper limit functions. J. Inequal. Appl. $2020,5(2020)$

37. Huang, X.S., Luo, R.C., Yang, B.C.: On a new extended half-discrete Hilbert's inequality involving partial sums. J. Inequal. Appl. 2020, 16 (2020)

38. Yang, B.C., Wu, S.H., Chen, Q.: On an extended Hardy-Littlewood-Polya's inequality. AlMS Math. 5(2), 1550-1561 (2020)

39. Liao, J.Q., Wu, S.H., Yang, B.C.: On a new half-discrete Hilbert-type inequality involving the variable upper limit integral and the partial sum. Mathematics 8, 229 (2020) https://doi.org/10.3390/math8020229 
40. Yang, B.C., Huang, M.F., Zhong, Y.R.: Equivalent statements of a more accurate extended Mulholland's inequality with a best possible constant factor. Math. Inequal. Appl. 23(1), 231-244 (2020)

41. Rassias, M.T.H., Yang, B.C.: A reverse Mulholland-type inequality in the whole plane with multi-parameters. Appl. Anal. Discrete Math. 13(1), 290-308 (2019)

42. Rassias, M.T.H., Yang, B.C.: On a Hardy-Hilbert-type inequality with a general homogeneous kernel. Int. J. Nonlinear Anal. Appl. 7(1), 249-269 (2016)

43. Rassias, M.T.H., Yang, B.C., Raigorodskii, A.: On Hardy-type integral inequalities in the whole plane related to the extended Hurwitz-zeta function. J. Inequal. Appl. 2020, 94 (2020)

44. Rassias, M.T.H., Yang, B.C., Raigorodskii, A.: On the reverse Hardy-type integral inequalities in the whole plane with the extended Riemann-zeta function. J. Math. Inequal. 14(2), 525-546 (2020)

45. Rassias, M.T.H., Yang, B.C.: On Hilbert-Type and Hardy-Type Integral Inequalities and Applications. Springer, Berlin (2019)

46. Rassias, M.T.H., Yang, B.C.: On an equivalent property of a reverse Hilbert-type integral inequality related to the extended Hurwitz-zeta function. J. Math. Inequal. 13, 315-334 (2019)

47. Kuang, J.C.: Applied Inequalities. Shangdong Science and Technology Press, Jinan (2004)

48. Kuang, J.C.: Real Analysis and Functional Analysis (Continuation) (Second Volume). Higher Education Press, Beijing (2015)

49. Wang, Z.Q., Guo, D.R.: Introduction to Special Functions. Science Press, Beijing (1979)

50. Faye Hajin Coyle, F. M.: Calculus Course, vol. 2, p. 397. Higher Education Press, Beijing (2006)

\section{Submit your manuscript to a SpringerOpen ${ }^{\circ}$ journal and benefit from:}

- Convenient online submission

- Rigorous peer review

- Open access: articles freely available online

- High visibility within the field

- Retaining the copyright to your article

Submit your next manuscript at $\boldsymbol{~ s p r i n g e r o p e n . c o m ~}$ 